\title{
Introducción a la traducción de la Nueva Deducción del Derecho Natural de Schelling
}

Faustino Oncina Coves

\section{(2) OpenEdition}

\section{Journals}

Edición electrónica

URL: http://journals.openedition.org/ref/403

DOI: $10.4000 /$ ref.403

ISSN: 2258-014X

Editor

EuroPhilosophie Editions

Referencia electrónica

Faustino Oncina Coves, «Introducción a la traducción de la Nueva Deducción del Derecho Natural de Schelling », Revista de Estud(i)os sobre Fichte [En línea], 3 | 2011, Publicado el 01 febrero 2011, consultado el 24 septiembre 2020. URL : http://journals.openedition.org/ref/403 ; DOI : https:// doi.org/10.4000/ref.403

Este documento fue generado automáticamente el 24 septiembre 2020.

(c) EuroPhilosophie 


\title{
Introducción a la traducción de la Nueva Deducción del Derecho Natural de Schelling
}

\author{
Faustino Oncina Coves
}

\section{NOTA DEL AUTOR}

Este trabajo ha surgido en el marco del proyecto de investigación "Hacia una Historia Conceptual comprehensiva: giros filosóficos y culturales" (FFI2011-24473) del Ministerio de Ciencia e Innovación, y fue ultimado durante una estancia en el Zentrum für Literatur- und Kulturforschung y en la Technische Universität de Berlín merced a una ayuda del Vicerrectorado de Investigación y Política Científica de la Universitat de València. Quiero manifestar mi agradecimiento a mis anfitriones en Berlín: Ernst Müller, Falko Schmieder y Thomas Gil.

1 Con ocasión del Primer Congreso Latino-americano sobre el pensamiento de J. G. Fichte, celebrado en la Universidad Federal de Goiás de Goiânia (Brasil) del 7 al 11 de noviembre de 2011, gracias a los buenos oficios de Hans-Christian Klotz, Thiago S. Santoro y Emiliano Acosta, los aforismos iusnaturalistas de Schelling tuvieron un, siquiera para mí, insospechado protagonismo. Varias ponencias los abordaron monográfica o episódicamente, agasajándolos ora en los relajados rellanos entre las secciones, ora en el fragor del debate que sucedía a las conferencias. Emiliano Acosta, Ana Carrasco Conde, Vicente Serrano (flamante premio Anagrama de Ensayo de ese mismo año), Felipe Schwember, Luciano C. Utteich..., cortejaban con alborozado rigor un prieto escrito que apenas lo leí se granjeó mi interés de tal manera que me vi compelido a traducirlo al castellano hace ya casi dos décadas. Durante años pensé para mi coleto que la cohorte de estudiosos schellinguianos, especialmente los hispanohablantes, lo habían pasado en silencio. Sin embargo, la insólita pujanza que ahora evidencia en nuestro cenáculo corrobora algo que esta era, sin resuello por las prisas y adicta al frívolo zapeo, parece haber olvidado: la investigación en ciencias 
humanas requiere una lenta y laboriosa digestión, esto es, tiempo. La cordial insistencia de los coordinadores de este número me ha animado a sumarme a su iniciativa con la reproducción de la versión de la Nueva deducción del derecho natural (NDDN) que en su día vio la luz en la revista Thémata (purgada de algunas erratas) y con esta Introducción, tributaria de la de entonces (titulada "Aporías y retos").

2 El punto de arranque de mi dedicación a Schelling fue mi tesis doctoral Derecho Natural y Revolución: La polémica de la filosofía política en la época de Fichte (1988). La primacía concedida en ella a Fichte obedecía a un doble motivo. En primer lugar, el Kant asumido por el Idealismo y el Romanticismo es el Kant fichteano; pero, paradójicamente, Fichte era el autor menos conocido y peor tratado de la pléyade idealista; en segundo lugar, en él confluyen una constelación de pensadores que han tenido una presencia casi anónima en la historia de las ideas a pesar de su extraordinaria impronta en ese período. No empleo gratuitamente el término "constelación", porque estaba familiarizado con el enfoque de Dieter Henrich" ${ }^{1}$. Desde enero de 1989 trabajé en el Kant-Archiv de la Universidad de Maguncia en colaboración con su director Rudolf Malter y con el del Studium Generale, Otto Saame, en un proyecto de investigación sobre la influencia de la Revolución Francesa en la aetas kantiana. A partir de 1993 frecuenté el Instituto MaxPlanck de Historia del Derecho Europeo de Fráncfort del Meno con el aval de Michael Stolleis y Heinz Mohnhaupt. Este eje Valencia-Maguncia-Fráncfort del Meno fue decisivo para que, en unas óptimas condiciones para tal empresa, acariciara el plan de preparar una antología de filosofías poskantianas del derecho, un plan nunca del todo consumado y ahora en barbecho, aunque he ido desgranando de modo disperso algunos resultados relacionados con Erhard ${ }^{2}$, Maimon ${ }^{3}$, Schelling y Fichte ${ }^{4}$. En lista de espera estaba Paul Johann Anselm Feuerbach ${ }^{5}$. Por consiguiente, en este ambicioso y descabalado plan se encuadraba la edición crítica de la NDDN de Schelling ${ }^{6}$.

3 Las claves que me condujeron a las contribuciones del jacobino Erhard, del judío errante Maimon y del joven aún desclasado académicamente Schelling las ofrece el propio Fichte, quien afirma explícitamente que fueron ellas las que le impulsaron a reelaborar las ideas que había expuesto en los denominados Escritos de Revolución ${ }^{7}$ de 1793 y a dar el salto, apoyado sobre una nueva plataforma, la de la Doctrina de la Ciencia, al fructífero quinquenio de Jena (1794-1798). El titán de Jena, como lo bautizó Hölderlins, hizo de zahorí señalando él mismo, de entre la abigarrada plétora de publicaciones sobre el tema, las fuentes que le inspiraron en su filosofía práctica ${ }^{9}$, en su Derecho Natural (1796-1797) y en su Ética (1798).

4 La NDDN continúa sumida en un cierto ostracismo por partida doble: por un lado, ha quedado desplazada a los márgenes del currículum filosófico de su autor o simplemente ninguneada; por otro, da la impresión de ser un eslabón perdido en las historias del iusnaturalismo. Su publicación troceada en dos partes y demorada por los editores ha contribuido a rebajarlo a un cuerpo extraño en la obra de Schelling, a pesar de que éste consideraba muy innovadoras las ideas que allí expresaba ${ }^{10}$. A pesar de la valoración altamente positiva que el artículo les mereció a los editores del Philosophisches Journal como confirma su nota al comienzo de la segunda parte de la NDDN-, la caricatura que de él ofreció Friedrich Nicolai, uno de los mandarines de la filosofía popular, con el burlesco título El Yo que se pone a sí mismo del licenciado Sr. Schelling o Nueva deducción del derecho natural (1798), tampoco coadyuvó a su exitosa recepción ${ }^{11}$. $Y$, sin embargo, es su única incursión monográfica en este campo, a diferencia de sus compañeros de generación, asiduos agrimensores del terreno de la filosofía del derecho. Tal 
efervescencia no es ajena a la furia constitucionalista que siguió a la Revolución Francesa y a las tentativas de codificación del corpus prusiano -lo que se ha llamado reforma desde arriba-. El impacto de esta doble circunstancia (la revolución del pueblo y la reforma no precipitada) en el orbe del criticismo se traduce en una hiperactividad entre los kantianos -que contrasta con el silencio de su inspirador, que sólo se pronunciará de forma sistemática en la Metafísica de las costumbres de 1797, si bien había dejado una ristra de indicios en escritos previos-. Entre sus filas cabe distinguir una primera generación de reputados juristas como G. Hufeland y Th. Schmalz (amén de filósofos consagrados como K. L. Reinhold), a cuyo remolque van los Escritos de Revolución fichteanos, y una segunda más amplia y polifónica, particularmente prolífica a partir de 1795: S. Maimon, J. Ch. Reinhard, J. B. Erhard, P. J. A. Feuerbach, Ch. E. Schmid, J. Ch. Hoffbauer, K. H. Heydenreich, J. Ch. G. Schaumann..., que pondrá al descubierto los déficits de aquellos padres fundadores del criticismo jurídico: la reducción del derecho a la moral, la exaltación del individuo, el carácter problemático de la coacción y de la instancia que la ejerce... Todos ellos pugnan por encarnar, como ironiza Schelling, el "espíritu literal" del kantismo y ese prurito incrementó la productividad de sus delfines y acólitos. En marzo de 1795 un abrumado Forberg registraba esta hipertrofia: "En el espacio de tres años los kantianos han importunado al mundo con doce teorías del derecho natural, ni una menos, y la que hace trece va a aparecer muy pronto"12. La mejor cartografía del mapa jurídico en esta época la realizó P. J. Anselm Feuerbach en su densa Crítica del derecho natural como propedéutica para una Ciencia de los derechos naturales (1796), imbuida asimismo del ideario del "gran hombre" de Königsberg, como revela el mismo título. Los iusnaturalistas kantianos, distribuidos entre partidarios de la deducción absoluta o relativa del derecho a partir de la moral -o de una tercera vía ecléctica o sincrética-, incurrían en el error de erigir la ley moral en la fuente del derecho -definido como un estar permitido (Dürfen o Erlaubtsein)-. De este lapsus principal se siguen, consecuentemente, una serie de carencias: "1) No es posible ningún derecho externo. 2) No es posible ninguna distinción entre el derecho natural y la moral. 3) El derecho, que debe ser algo dado y producido por la razón (conectado positivamente con ella), se convierte en una mera negación, en algo conectado sólo negativamente con la razón, y es confundido con lo justo. 4) No es posible ninguna libertad jurídica externa. 5) Es imposible una demostración para los derechos de coacción"13. En semejante caldo de cultivo brota el Fundamento del derecho natural de Fichte.

$5 \quad$ Por otro lado, los rescoldos del debate entre Lessing y Jacobi sobre el espinosismo -el primero vindicándolo, el segundo estigmatizándolo- no se han apagado en el período de gestación de la NDDN, sino que persiste la espinosamanía, si bien se alternan e incluso cohabitan las filias y las fobias. Fichte es un buen ejemplo. Describe en 1794 la parte teórica de su Wissenschaftslehre como un espinosismo sistemático, esto es, formalmente monista ("La parte teórica de nuestra Doctrina de la Ciencia, desarrollada a partir de los dos últimos principios, dado que aquí el primero sólo tiene un valor regulativo, es efectivamente, como se mostrará a su tiempo, el espinosismo sistemático", GWL, GA I, 2, 282), y un recensor del Escrito de justificación lo tacha de "espinosismo invertido" (umgewandter) (GA I, 6, 14), al igual que hará Jacobi en su célebre carta de marzo de 1799 tras recibir el 18 de enero la Apelación ${ }^{14}$. En la ilación de aforismos que componen el artículo, Schelling sigue bajo el embrujo espinosista ${ }^{15}$. Ellos propulsan la implosión del derecho natural poskantiano y del iusnaturalismo. Siguiendo a Norberto Bobbio, se entiende por iusnaturalismo la revitalización, desarrollo y difusión que la idea del 
derecho natural experimentó durante la edad moderna, entre comienzos del siglo XVII y finales del XVIII. El método racional que emplean sus representantes ha de permitir la reducción del derecho y la moral (así como de la política) a ciencia demostrativa y el único principio de legitimación de las sociedades políticas es el consentimiento, por lo que la sociedad civil se concibe como una asociación voluntaria de individuos basada en un contrato ${ }^{16}$. La paradoja estriba en que Schelling se vale de la estrategia demostrativa -habitual entre los iusnaturalistas- para acometer la demolición del derecho natural y de uno de sus elementos nucleares, la convención.

6 El contrato, tras su momento de esplendor con la Ilustración, inicia una irredimible agonía -Rawls derogará el anatema que pesaba sobre él-. El primer Fichte y su séquito de exégetas de todo color ya auguraban nolens volens malos presagios para el iusnaturalismo, pero Schelling entona su réquiem. La apología fichteana del pacto en 1793 es tan expugnable que ofrece fácil abono a cualquier antipactista. Schelling es un precursor del demoledor ataque hegeliano contra el contractualismo. Los motivos esgrimidos para justificar la extinción política del contrato son contundentes: con su recurso, además de incurrir en un regressus ad infinitum al intentar elucidar su necesidad como instancia fundadora del Estado, la sociedad acaba siendo engullida por la naturaleza, desapareciendo las marcas distintivas de ambas. Fichte establece precozmente una provocadora simetría entre revolución y contrato. En los Revolutionsschriften el contrato equivale a un compromiso de continuo revocable por cualquiera de los contratantes, lo cual conduce a una iteración de infinitos contratos y a un permanente transfuguismo entre el estado civil y el estado natural. El arbitrio es el único canon de la ciudadanía. La repercusión ${ }^{17}$ de estos Escritos ratifica el inminente declive del contrato a pesar de su asténica redención en el Fundamento del Derecho Natural.

7 En la NDDN Schelling divide el itinerario de su argumentación en tres episodios: la moral, la ética y el derecho. El primero lo protagoniza la moral, donde el sujeto libre aspira al infinito en una acción infinita: "§ 4 esfuérzate por ser absolutamente libre, esfuérzate por someter a tu autonomía toda potencia heterónoma". Esta aspiración, enarbolada imperativamente por todos los individuos, desencadena el conflicto entre las voluntades morales: "§ 7 Me proclamo señor de la naturaleza y exijo que esté determinada enteramente por la ley de mi voluntad. [...]. El mundo entero es mi propiedad moral". La pluralidad de voluntades morales, candidatas todas a la dominación absoluta de la tierra, aboca ineluctablemente a la fricción: "§ 21 Luego la causalidad incondicionada de los seres morales deviene conflictiva en el esfuerzo empírico y comienzo a oponer mi libertad a la libertad de todos los demás".

8 En el segundo acto la ética configura a la vez un ámbito de reconocimiento limitativo y de transgresión de los límites. La ética, que vive en la tensión del antagonismo entre los iguales, no renuncia, sino que se entrega a abrir flujos de comunicación y cooperación. Discordia y concordia se abrazan aquí, la insociable sociabilidad anida justamente en este episodio: "§ $45 \mathrm{El}$ mandato supremo de toda ética reza así: [...] obra de tal modo que mediante tu acción (según su contenido y su forma) ningún ser racional sea puesto como mero objeto, sino como sujeto cooperante«.

9 La ética de la concordancia ( $\$ 43$ ), en su forcejeo con la moral ególatra, pretende arrojar luz sobre la noche del Terror jacobino, para enseñar cómo la infatuación del individuo, moralmente legitimada, aniquila toda alteridad, toda discrepancia. La vocación moral del Yo bendice la individualidad omnímoda. El sujeto formador debe ser 
para el Fichte de 1793 el legítimo propietario, no el sujeto heredero aferrado a los privilegios legados ${ }^{18}$. El fundamento de la propiedad reside en la forma que un cierto individuo le imprime a una cierta materia. Desde el punto de vista de la materia, todo hombre posee originariamente un derecho de apropiación sobre la tierra entera. Desde el punto de vista de la forma, todo hombre es propietario, es decir, tiene un derecho real de propiedad, en función del trabajo (Formation, Arbeit, Bearbeitung) que ha invertido para elaborar la materia bruta; en suma tiene un derecho de propiedad sobre la materia modificada por él (GA I, 1, 269).

10 Schelling atisba el peligro que entraña la apoteosis virtuosa del individuo. La ipseidad (Selbstheit) de la moral es compensada ahora con el reino de los seres morales exigido por la ética (\$ 31). La ebriedad moral de infinitud y la sobriedad ética de las finitudes precisan una síntesis allende ambas dimensiones. La dialéctica entre el primer jalón, en el que el sujeto aspira a apropiarse de todas las cosas, y el segundo, en el que ha de limitar su voluntad posesiva en cuanto ser finito que concibe a los otros como sus límites y provistos de ese mismo afán de posesión, remite a una tercera dimensión, el derecho ${ }^{19}$.

11 La coacción y el contrato son dos fórmulas con miras a conjurar los dilemas de atracción y repulsión, cooperación y colisión imperantes en la ética. Pero ambos expedientes se revelan inoperantes para mantener a los sujetos dentro de sus radios de acción. La tendencia infinita hacia el dominio del mundo, la avidez acaparadora jaleada por la autonomía moral torna el contrato un artilugio jurídico fatalmente lábil. El objeto del contrato, la materia del mundo, no es, en último extremo, susceptible de pacto, pues la voracidad del individuo no acaba nunca de saciarse, y "para asegurar un contrato sería necesaria una serie infinita de contratos, de los que cada uno confirmaría el precedente, pero él mismo estaría a su vez necesitado de una nueva confirmación" ( $\$$ 85). La interpretación fichteana del imperativo moral no es sino un mero revestimiento del egoísmo del individuo, quien, para acreditarse como tal, reivindica un contrato infinitamente revisable, esto es, revocable. La materia de la voluntad, el mundo entero, es algo indefinido, pues aquello de lo que cabe apropiarse, el objeto de la propiedad, no puede acotarse en un contrato puntual, porque siempre lo excede. Esa voluntad inextirpable de infinito, ese frenesí posesivo del sujeto, convierte el perpetuo conflicto en intrínseco al contrato, ya que siempre afloran nuevos objetos en litigio. El contrato no dirime, sino que magnifica y perpetúa la disputa. Con el recurso al contrato se pretende cumplir el mandato de la ética consistente en la concordancia intersubjetiva, pero la individualidad siente el acuerdo como una amenaza, como un modo de saciar definitivamente su sed insaciable de infinito. El contrato, incluso entendido en su máxima ductilidad, como infinitamente revisable, pone en jaque la soberanía del Yo, la autoafirmación del individuo; y la tentativa de menguarla sólo consigue evidenciar aún más la prístina ausencia de límites que le corresponde como ser moral: "§ $144 \mathrm{La}$ libertad, la forma originaria de la voluntad individual, debe retornar a su originaria ilimitación apenas está en juego su autoafirmación. Es potencia absoluta capaz de someter cualquier potencia que se le oponga".

12

El contrato no supone una escapatoria a la contradicción entre cooperación y oposición con miras a la aceptación de individualidades idénticas, al respeto recíproco. El contrato ya no es fundamento de la coacción, sino un perenne choque entre fuerzas ( $\$$ 149). Repudiado el contrato, la coacción resta como único medio para garantizar el 
reconocimiento mutuo de las individualidades; pero la coacción se manifiesta como coacción física, como imposición por la fuerza bruta, e ignora, una vez comienza a desplegarse, la esencia moral, racional, de los sujetos, al reificarlos. La coacción vuelve indiscernibles sociedad y naturaleza, reduce el campo social a un campo natural, magnético, donde posee mayor fuerza de atracción quien dispone de una mayor fuerza física.

13 El artículo desemboca en la urgencia de nuevos escenarios y en el anacronismo de la Ilustración, sea bajo su forma sosegada, republicana, sea bajo la agitada, revolucionaria, pues el derecho natural se funde con el derecho según leyes naturales: "El derecho natural se destruye necesariamente él mismo en sus consecuencias (en tanto que deviene derecho de coacción), esto es, suprime todo derecho. En efecto, es a la superioridad física a la que se confía en último extremo la conservación del derecho" (§ 162). La coacción arruina la vigencia de la criteriología de lo humano, de un álter ego. Schelling había hecho del descubrimiento de la otredad la cumbre de la transición de la moral a la ética: "§ 13 Donde mi potencia moral encuentra resistencia, ya no puede haber naturaleza [...]. ¡Aquí está la humanidad! [...]. § 15 En cuanto siento limitada mi libertad, reconozco que no estoy solo en un reino de seres morales a quienes corresponde, a todos ellos, esta misma libertad sin límites".

14 El corolario del razonamiento afecta decisivamente a los derroteros que va a seguir la filosofía política del idealismo y encauza el devenir del propio Schelling ${ }^{20}$. El derecho se disuelve en nuda coacción y acaba remontándose a un estadio previo de violencias físicas pugnando entre sí, a una mecánica de fuerzas concurrentes, cosificando el mismo mundo jurídico como requisito indispensable para propiciar un paso ulterior metajurídico. La resolución de las aporías emanadas del precoz fichteanismo precisa un tramo postrero, apenas bosquejado: "Por consiguiente, el derecho natural conduce necesariamente a un nuevo problema: el de hacer idéntica la potencia física del individuo a la potencia moral del derecho, es decir, al problema de encontrar un estado en el que la fuerza física siempre esté del lado del derecho. En cuanto nos ocupamos de la solución de este problema, entramos en el terreno de una nueva ciencia" (\$ 163).

15 En el Sistema del idealismo trascendental (1800) se hace eco de dos conclusiones de sus aforismos. En primer lugar, el derecho se desprende del lastre pernicioso de la moralidad, pues su alianza ha engendrado un tenebroso despotismo. En segundo lugar, el Estado aparece así como una máquina antirrevolucionaria, que no busca su legitimación en el derecho. La maquinaria del Estado controla con la lógica del resorte tanto la fuerza del impulso egoísta como la de la insurrección ${ }^{21}$.

16 Estamos en las antípodas no sólo del Fichte jacobino, sino también del Fichte de Jena, quien, consciente de las tachas de aquél y resuelto a enmendarlas, no cohonesta prima facie el epitafio del iusnaturalismo. Para garantizar la realización sensible del derecho es menester que la ley sea asumida por un poder, por el poder supremo. No hay eficacia real del derecho sin un Estado. La voluntad general no sólo será voluntad del derecho, sino que también será preponderante al representar la voluntad de la comunidad, y, en consecuencia, superior a cualquier voluntad injusta ${ }^{22}$. El contrato social es caracterizado como un contrato de propiedad, de fijación de límites de las propiedades de los ciudadanos a fin de no entrometerse en la esfera de acción ajena. Estos límites de la libertad exclusiva de cada uno han de ser protegidos no mediante la invocación a la buena voluntad -por eso el derecho no se puede deducir de la moral-, sino por la fuerza física de la coacción. Esto comporta una nueva modalidad de contrato, el contrato de 
protección (GA I, 4, 8-10). La voluntad general material, que antes era meramente negativa, la renuncia a inmiscuirse en la esfera del otro, la mera abstención u omisión, se convierte en afirmativa, en una prestación positiva, la defensa de los bienes ajenos.

17 La fusión de los individuos en un totum se realiza bajo el efecto del azar imprevisible de quién será el protector o el protegido. Este contrato que troca los dos anteriores en un contrato social se llama contrato de unión. El triádico contrato de ciudadanía (Staatsbürgervertrag), con sus afluentes de propiedad, protección y unión, sirve para esquematizar la soberanía de la voluntad general como momento regenerador de la sociedad política ${ }^{23}$. Pero es del eforato, órgano supervisor del ejecutivo y convocante de la comunidad, de quien depende la seguridad del todo, la parusía fenoménica de la libertad (GA I, 3, 328, 442, 453, 459). Hegel y Schelling pusieron de relieve las fisuras de ese dispositivo, vinculado al contractualismo. Mediante los éforos se auspicia una escisión de la voluntad general para velar por que actúe como tal. El poder ejecutivo y el eforato aparecen así como dos representaciones de la voluntad general, y con esa escisión se pretende hacer prevalecer su pureza, su rectitud; mas es el primer poder quien detenta de modo exclusivo la coacción, y le resultaría sencillo paralizar el desempeño de las funciones del contrapoder, la convocatoria del pueblo y su labor de supervisión. Si el eforato vigila al ejecutivo para lograr una práctica más justa de ese poder, entonces la suprema justicia se alcanzaría multiplicando los contrapoderes. La realización del derecho se salda con un regressus ad infinitum ${ }^{24}$. Es el infinito malo que ya Schelling le imputaba al frágil contractualismo del joven Fichte. Sólo la violencia policial es capaz de imponer la voluntad general, de enlazar las voluntades individuales con una libertad universal. La aplicación de la fuerza exterior y el control rígido y ubicuo por el Estado es el único medio de ligar los átomos inertes que componen el pueblo, de construir artificialmente la totalidad. El contractualismo y el eforato no son sino recursos fallidos para aproximar los elementos escindidos e impiden concebir la totalidad política como un organismo que integra la voluntad del individuo y la de la comunidad.

18 Aflora el trauma causado por la impotencia de los arbitrios para su entendimiento recíproco, llevado a su paroxismo por la Revolución Francesa. La metáfora mecanicista de Schelling en el Sistema no debe ofuscarnos, ya que es un dislate hermenéutico franquear el hiato entre el Estado real y el Estado ideal, confundiendo una etapa coyuntural con el acabamiento de la historia ${ }^{25}$. El Estado máquina pertenece a los dominios del primero, tan necesitado de la coacción, mientras que ésta es ajena al organicismo propio del régimen auténtico, donde parte y todo, ciudadano y Estado son al unísono medio y fin. Schelling se vale de un motivo del Juicio Reflexionante que pretende salvar la contingencia categorial de los seres organizados -motivo que Kant mismo convierte en analogía política ${ }^{26}$, pero para traspasar las prevenciones del criticismo y del fichteanismo ${ }^{27}$, que siempre le entregan el crédito legitimador al contrato -sea al originario o al de ciudadanía-.

19 En la NDDN Schelling se enfrenta al reconocimiento del otro con letra fichteana y espíritu antifichteano. Priva al Yo de su facultad generadora de una doctrina del derecho. Aquí todavía no ha podido aquilatar, aunque lo hará en el Sistema con un veredicto negativo, el tránsito de los Escritos de Revolución al Fundamento del Derecho Natural. La NDDN demuestra el fiasco del iusnaturalismo erigido sobre un Yo individual pletórico que acaba siendo víctima de su propia telaraña de ambición. El contrato constituye un falso punto de sutura entre la moral de conquista del Yo y el afán ético de 
cooperación. A diferencia de Fichte, en Schelling no reside en el derecho la clave de la síntesis entre la libertad y la naturaleza, sino que depende de otra ciencia, de una nueva ciencia - ¿la física o filosofía de la naturaleza?, ¿la historia, más capaz de hacer calar vía mito la concordancia en la oposición, la universalidad en la individualidad?--

Finalmente, deseo agradecer a Thémata. Revista de Filosofía y a su director, Jacinto Choza, su autorización para incluir aquí la versión que inicialmente apareció en el número 11, correspondiente al año 1993, de dicha publicación. Aunque sin duda esta versión -y por tanto toda traducción- es mejorable, he preferido retocarla lo menos posible y limitarme a la criba indicada al comienzo.

\section{NOTAS}

1. La teoría de las constelaciones -un método de investigar la concurrencia de autores diferentes en un espacio acotado de pensamiento común, que halla su vergel en el rastreo de la génesis del temprano Idealismo alemán- ha acreditado su fecundidad al destacar el valor intrínseco de este florilegio de casi ignotas aportaciones -ignotas por estar a la sombra de la gigantomaquia de los grandes nombres de la época- y al descubrir sus nexos ocultos ("missing links"), también con las figuras cumbre, pero le acecha la amenaza de incurrir en una irreflexiva doxografía y en una suerte de endogamia conceptual autocomplaciente, que, pese a los buenos resultados logrados, se me antoja cada vez más estéril si no se amplía su campo de visión. o dicho de otra manera, su rendimiento refulge dentro de los confines del idealismo, mas arrostra el peligro de una cierta estrechez de miras envanecida, porque mira de soslayo tanto lo anterior como lo posterior, o simplemente lo fagocita. Había conceptos centrales (derecho y contrato, vg.), que, restrospectiva o prospectivamente, rompían las costuras de esa constelación. Además, no es fácil, amén del caso emblemático del idealismo alemán, hallar en el pasado otros ejemplos de constelaciones. Las traducciones y ediciones aludidas -junto al crucial asesoramiento de Heinz Mohnhaupt, coautor de la voz "constitución" en el célebre macrodiccionario Conceptos históricos fundamentales de Reinhart Koselleck- me indujeron a superar (en el sentido hegeliano) el método de las constelaciones mediante la historia conceptual, con la que me he codeado estos últimos años. El opus magnum del método de D. Henrich es el libro Grundlegung aus dem Ich: Untersuchungen zur Vorgeschichte des Idealismus: Tübingen-Jena (1790-1794), Fráncfort del Meno, Suhrkamp, 2004. Cf. D. HENRICH, Konstellationen. Probleme und Debatten am Ursprung der idealistischen Philosophie (1789-1795), Stuttgart, Klett-Cotta, 1991; M. MULSow, M. STAMM (eds.), Konstellationsforschung, Fráncfort del Meno, Suhrkamp, 2005.

2. J.B. Erhard. Apología del diablo. Introducción, traducción del alemán, notas y estudio bibliográfico, Sevilla, Editorial Er, 1993.

3. "Salomon Maimon: Sobre los primeros fundamentos del derecho natural" (1795). Introducción, traducción y notas, en: Revista venezolana de filosofía (Caracas), 27 (1992), pp. 89-129.

4. “J. G. Fichte. Reseña de Hacia la paz perpetua de Kant”. Introducción, traducción y notas, en: Daimon (Murcia), 9 (1994), pp. 373-381; J. G. Fichte. Fundamento del derecho natural según los principios de la Doctrina de la Ciencia. Traducción del alemán (en colaboración con J. L. Villacañas y M. Ramos) y notas, Madrid, Editorial Centro de Estudios Constitucionales, 1994; J. G. Fichte. Algunas lecciones sobre el destino del sabio. Introducción, traducción, comentarios y notas (en colaboración con M. Ramos), Madrid, Istmo, 2002. 
5. Me refiero a su Ensayo sobre el concepto de derecho, aparecido en el Philosophisches Journal en 1795, que constituye un esbozo de un libro crucial: Crítica del derecho natural como propedéutica para una ciencia de los derechos naturales (1796).

6. “F.W.J. Schelling. Nueva deducción del derecho natural". Introducción, traducción y notas, en: Thémata. Revista de Filosofía (Sevilla), 11 (1993), pp. 217-253.

7. Un botón de muestra lo encontramos en su panfleto Reivindicación de la libertad de pensamiento (1793) y en la segunda edición ampliada del Ensayo de una crítica de toda revelación (1793), disponibles en castellano (la primera en Tecnos, Madrid, 1986, y la última en Biblioteca Nueva, Madrid, 2002). Sus Contribuciones destinadas a rectificar el juicio del público sobre la Revolución Francesa (1793) siguen la senda marcada por los dos libros anteriores.

8. "Hölderlin me escribe de vez en cuando desde Jena. [...] Asiste a los cursos de Fichte y habla entusiasmado sobre él como de un titán que lucha por la humanidad y cuyo ámbito de influencia ciertamente no se limitará al interior de las paredes del auditorio" (Carta de Hegel a Schelling de fines de enero de 1795, en: J.A. DÍAZ (ED.), CORRESPONDENCIA. KANT, FICHTE, SCHELLING, HEGEL. TRADUCCIÓN, INTRODUCCIÓN Y NOTAS DE HUGO OCHOA DISSELKOEN Y RAÚL GUTIÉRREZ. BOGOTÁ, UNIVERSIDAD NACIONAL DE COLOMBIA, 2011, P. 304).

9. En su Fundamento del Derecho Natural de 1796 leemos: “Con excepción de algunos indicios excelentes del Sr. Dr. Erhard, en varios de sus escritos más recientes [en la página anterior cita expresamente su Apología del diablo], y del Sr. Maimon, en un artículo sobre el derecho natural en el "Philosophisches Journal" del Prof. Niethammer, el autor de este escrito todavía no había encontrado ningún atisbo de que un filósofo desconfiara de la manera habitual de tratar el derecho natural" (GNR, GA I, 3, 323; ed. esp. Centro de Estudios Constitucionales, Madrid, 1994, p. 112). En su Ética de 1798, en el marco de la inferencia de la libertad ajena, elogia el tino del aforismo de la NDDN (§ 13) que deslinda la doble faceta de la resistencia o limitación, ora como naturaleza, ora como humanidad, esto es, como álter ego: “Acertadamente lo expresa el señor Schelling [...]” -a continuación cita casi literalmente el § 13 (SSL, GA I, 5, 204; ed. esp. de Jacinto Rivera de Rosales: Ética o El sistema de la doctrina de las costumbres según los principios de la Doctrina de la Ciencia, Madrid, Akal, 2005, p. 260).

10. La NDDN fue probablemente concebida en 1795 y culminada a comienzos de 1796, año en que apareció su primera parte en el Philosophisches Journal; la segunda se publicó el año siguiente. Consta de 163 parágrafos o aforismos (HKA I, 3, 139-175). Véanse la citada nota que los editores incluyeron en la segunda entrega de estos aforismos ("Acerca de la relación de esta deducción con recientes investigaciones sobre este mismo asunto, se encontrará una declaración del autor al final del artículo. Por nuestra parte, tan sólo debemos indicar que este escrito nos fue enviado hace ya un año y medio, y que, por sus originales opiniones, incluso tras la aparición de nuevos trabajos, no podemos considerarlo en absoluto obsoleto", HKA I, 3, 157) y el Post Scriptum del propio autor ("Los presentes aforismos no aspiran a ser más que aforismos. Su comentario se lo reserva el autor tanto más cuanto que las recientes contribuciones del derecho natural, que no ha podido utilizar para este trabajo, le proporcionarán abundante material con miras a reflexiones más maduras así como múltiples ocasiones para desarrollar sus principios de una forma más completa", HKA I, 3, 175). Schelling fue pronto consciente de las novedades que ofrecía su NDDNsu carta a Niethammer del 23 de marzo de 1796 es muy elocuente ("Quizá le presente para su publicación en el Philosophisches Journal unos aforismos que he redactado para mis clases sobre Derecho Natural, y que contienen, según me precio, una nueva deducción del Derecho Natural, más satisfactoria que las efectuadas hasta el momento. Hubiera querido mandárselos ya con esta carta [...] pues no me gustaría que se me adelantase otro y se creyese que yo lo había plagiado")y de que se encontraba en los umbrales de un giro en la filosofía del derecho, en el que participarían otros coetáneos (dos magníficos testimonios a este propósito los constituyen el libro de P. J. A. Feuerbach Crítica del Derecho Natural de 1796 y la reseña de los primeros volúmenes del Philosophisches Journal que Friedrich Schlegel preparó en 1797 para la Allgemeine Literatur- 
Zeitung). Coincido con los responsables de la edición histórico-crítica cuando sugieren que Schelling deseaba anticiparse a Fichte (sin descartar tampoco a Hufeland), pues, tras las acertadas objeciones que le plantearon desde varios frentes a sus Escritos de Revolución y al descubrimiento de la Doctrina de la Ciencia, cabía esperar un próximo ensayo fichteano sobre este tópico (cf. HKA, I, 3, 125). Además, es plausible que Hölderlin, antes de abandonar Jena, informase a sus amigos de que en el semestre de verano de 1795 Fichte había anunciado un curso sobre derecho natural, al que tuvo que renunciar ante la agitación imperante en la ciudad y su retiro a la cercana villa de Osmannstadt. El editor de la obra crítica del último, R. Lauth, no duda en señalar a éste como el aludido en la misiva de Schelling (Die Entstehung von Schellings Identitätsphilosophie in der Auseinandersetzung mit Fichtes Wissenschaftslehre (1795-1801), Friburgo, Alber, 1975, pp. 36-37).

11. El colofón de ese libelo antiidealista reza así: “¡Roguemos para que, en lugar de la filosofía abstrusa y ampulosa, impere universalmente la sana razón en la literatura alemana! ¡Amén!” (Leben und Meinungen Sempronius Gundibert's eines deutschen Philosophen. Nebst zwei Urkunden der neuesten deutschen Philosophie, 1798, p. 342). Entre los escasos exégetas del NDDN merecen especial mención: A. HOLLERBACH, Der Rechtsgedanke bei Schelling. Quellenstudien zu seiner Rechts- und Staatsphilosophie, Fráncfort del Meno, Klostermann,1957; M. OSTEN, Der Naturrechtsbegriff in den Frühschriften Schellings, Colonia, 1969; C. CESA, La filosofia politica di Schelling, Bari, Laterza,1969; J. RIVELAYGUE, "Schelling et les apories du droit", en: Cahiers de philosophie politique, 1 (1983), pp. 13-62; M. VICEDO, "Derecho Natural en el joven Schelling”, en: Quaderns de Filosofia i Ciencia, 9/10 (1986), pp. 453-456; J.L. VILLACAÑAS, "Mito y Estado. Esbozo de una teoría contrarrevolucionaria del Estado en el Schelling de Jena", en: AA.VV., Eticidad y Estado en el idealismo alemán, Natán, Valencia,1987, pp. 89-112; C. CESA, "Schellings Kritik des Naturrechts", en: Die Praktische Philosophie Schellings und die gegenwärtige Rechtsphilosophie, ed. de H. M. Pawlowski, s. Smid y R. SPECHT, Stuttgart-Bad Cannstatt, Frommann, 1989, pp. 177-197; F. ONCINA, "Schelling como catalizador de la crisis del iusnaturalismo", en: A. LEYTE COELLo (edit.), Una mirada a la filosofía de Schelling, Vigo, Universidade de Vigo, 1999, pp. 97-104; M. G. VATER, "Schelling's Aphorisms on Natural Right (1796/97): A Comparison with Fichte's Grundlage des Naturrechts", en: T. ROCKMORE, D. BREAZEALE (eds.), Rights, Bodies and Recognition. New Essays on Fichte's Foundations of Natural Right, Ashgate, Aldershot, 2006, pp. 195-211. En esta escueta bibliografía no hay ninguna pretensión de exhaustividad. En el 7. Internationaler Fichte-Kongress "Fichte/Schelling: der Idealismus in der Diskussion" celebrado en Bruselas de 2009 tenía previsto presentar la ponencia: "La necrológica schellinguiana del Derecho Natural: La transición de los Escritos de Revolución (1793) de Fichte al Fundamento del Derecho Natural (1796-97)", que alimenta en buena medida esta Introducción.

12. Citado en X. LÉON, FICHTE ET SON TEMPS, VOL. I, PARÍS, 1922, P. 473.

13. Kritik des natürlichen Rechts, Altona, 1796; reimp. Hildesheim, Olms, 1963, p.130. Otro balance de los trabajos en este ámbito a partir de 1795 -por lo tanto, recoge un intervalo de tiempo en parte omitido por el libro de Feuerbach- es la reseña que Friedrich Schlegel publicó en 1797 en la reputada Allgemeine Literatur-Zeitung en la que examina los artículos más sobresalientes sobre derecho natural aparecidos en el Philosophisches Journal, revista que abogaba programáticamente por la difusión y la consumación del criticismo como ciencia. Schlegel resume las tendencias incipientes en la filosofía jurídica que sintetiza en una cuádruple moda: "1) El principio del derecho es independiente de la moral; 2) no es sólo útil técnicamente, sino necesario práctica y absolutamente; 3) es la condición y limitación de una ley positiva; 4) la posibilidad de la ley jurídica se funda en el concepto de una comunidad de seres libres" (Kritische-Friedrich SchlegelAusgabe, edición a cargo de E. Behler, vol. 8, Múnich, 1975, p. 22). Fichte redactó, entre mayo y agosto de 1795, un esbozo de recensión, finalmente no concluida, titulada Para la reseña de los derechos naturales en el 'Journal' de Niethammer (GA II, 3, 395-406). 
14. "espinosismo invertido" (umgekehrten), en: F. H. JACOBI, CARTAS A MENDELSSOHN Y OTROS TEXTOS, BARCELONA, CÍRCULO DE LECTORES, 1996, P. 486. EN 1794 EL ESPINOSISMO MATERIAL LE PARECE AL PROPIO FICHTE LA EXPRESIÓN MÁS CONSECUENTE DEL DOGMATISMO FILOSÓFICO Y CONSTITUYE UNA FORMA INEQUÍVOCA DE "FATALISMO" (GWL, GA I, 2, 279-280, 310).

15. Los rastros de Espinosa en Schelling pueden seguirse a través de su correspondencia tubinguesa con Hegel: véanse las cartas de enero -en la que dice estar "trabajando en una ética à la Spinoza"- y febrero de 1795 -donde hallamos su famosa declaración "Entretanto, ¡me he convertido en spinozista!"- (J.A. DÍAZ (ED.), CORRESPONDENCIA. KANT, FICHTE, SCHELLING, HEGEL, PP. 300, 306-308; VOM ICH ALS PRINZIP, EN: HKA I, 2, 80; CARTA A NIETHAMMER DEL 22 DE ENERO DE 1796, EN: HKA III, 1, PP. 40-42). EN DEL YO COMO PRINCIPIO DE LA FILOSOFÍA (1795) HALLAMOS UNA OCURRENCIA DEL TÉRMINO DERECHO, PERO EXENTA DE ORIGINALIDAD Y EN PERFECTA SINTONÍA CON LA FILOSOFÍA JURÍDICA Y POLÍTICA ANTIEUDEMONISTA DE LA AETAS KANTIANA: “EL CONCEPTO DE DERECHO EN GENERAL, Y EL SISTEMA ENTERO DEL DERECHO NATURAL, SE BASA EN EL CONCEPTO DE LA POSIBILIDAD PRÁCTICA...; EL CONCEPTO DE DEBER, Y EL SISTEMA ENTERO DE LA ÉTICA, SE BASA, SIN EMBARGO, EN EL CONCEPTO DE LA REALIDAD PRÁCTICA". PARA EL SER FINITO EL DERECHO DEPENDE DEL DEBER Y “EL FIN SUPREMO HACIA EL CUAL TIENEN QUE APUNTAR TODAS LAS CONSTITUCIONES POLÍTICAS (QUE ESTÁN FUNDADAS EN EL CONCEPTO DE DEBER Y DERECHO) PUEDE SER ÚNICAMENTE LA IDENTIFICACIÓN DE LOS DERECHOS Y DEBERES DE CADA INDIVIDUO PARTICULAR" (HKA I, 2, 164-165). NO HAY UNA CORRESPONDENCIA ENTRE SCHELLING Y FICHTE QUE ABORDE DIRECTAMENTE LA NDDN (GRUNDLEGUNG UND KRITIK. DER BRIEFWECHSEL ZWISCHEN SCHELLING UND FICHTE 1794-1802, EN: FICHTE-STUDIEN, 25 (2005)). POR OTRO LADO, FICHTE ESTABA CONVENCIDO DE QUE DEL YO COMO PRINCIPIO DE LA FILOSOFÍA NO ERA SINO UN MERO COMENTARIO DE SU PROPIA OBRA (GA III, 2, 347) .

16. N. BOBBIO, "EL MODELO IUSNATURALISTA", EN: ESTUDIOS DE HISTORIA DE LA FILOSOFÍA: DE HOBBES A GRAMSCI, MADRID, DEBATE, 1985, PP. 73-149.

17. El trasfondo de la NDDN es un espectro heteróclito de autores (Spinoza, Schaumann, Flatt, Mendelssohn, Hufeland...), entre los cuales, empero, brilla Fichte, cuyas primeras obras, Ensayo de una Crítica de toda revelación (1792), Reivindicación de la libertad de pensamiento y Contribuciones destinadas a rectificar el juicio del público sobre la Revolución Francesa (1793), ya atraen la atención de los amigos del seminario tubingués -tal como prueban sus cartas a Hegel del 6 de enero, del 4 de febrero y del 21 de julio de 1795-. En la carta más antigua a Fichte que se conserva de Schelling, fechada en Tubinga el 26 de septiembre de 1794, a la que adjunta su breve escrito Sobre la posibilidad de una forma de la filosofía en general, le declara en un tono de rendido reconocimiento: "quisiera aprovechar esta ocasión para expresarle mi más sincero y profundo agradecimiento por lo que he aprendido a través de sus admirables obras" -obviamente, alude a las citadas arriba (J.A. DíAz (ed.), Correspondencia. Kant, Fichte, Schelling, Hegel, pp. 109, 300, 302, 306, 318).

18. "Sí, en efecto, no es posible otra manera de apropiación que mediante la formación; necesariamente todo lo que todavía no está formado, lo que todavía se encuentra en estado bruto, no es propiedad de nadie. Tenemos un derecho de apropiación sobre la materia bruta y un derecho de propiedad sobre la materia modificada por nosotros. El primero designa la posibilidad moral; el segundo la realidad moral y física" (GA I, 1, 268). Fichte defiende la teoría formativa de la propiedad, sin reparar en su lectura radical en clave de ejercicio de poder, de forma regiminis.

19. J. B. ERHARD DESCRIBE UNA TRAMA ANÁLOGA A LA QUE DENUNCIA SCHELLING, Y LLAMA LA ATENCIÓN SOBRE LAS MALIGNAS SECUELAS DE ESTE PRECOZ YO FICHTEANO: “LA ESCLAVITUD DE TODOS Y LA LIBERTAD DE UNO SOLO, LA CULTURA DE TODOS PARA LOS FINES DE ESTE ÚNICO INDIVIDUO” (GA I, 1, 252-253; VÉASE MI “INTRODUCCIÓN" A LA EDICIÓN DE LA APOLOGÍA DEL DIABLO). ERHARD Y SCHELLING DISENTIRÁN, NO OBSTANTE, EN EL DESENLACE. MIENTRAS QUE EL PRIMERO PIENSA HABER DESCUBIERTO EN EL DERECHO -NO EN EL CONTRATO- EL ANTÍDOTO CONTRA EL EGOÍSMO EXPANSIVO; EL SEGUNDO ACABA AFIRMANDO LA INEFICACIA DEL DISPOSITIVO JURÍDICO.

20. $Y$, sin duda, golpea mortalmente al criticismo, en cuya consumación porfiaba el idealismo: "Según el criticismo, mi destino consiste en el esfuerzo en pos de la ipseidad inmutable, de la libertad incondicionada, de la actividad ilimitada" (Cartas filosóficas sobre dogmatismo y criticismo, en: HKA I, 3, 106). En la Ética (1798) Fichte celebra estos parágrafos (en particular el § 13) de la NDDN por 
rescatar la vertiente dialógica del idealismo frente a la moral monológica que brotaba del imperativo categórico (SSL, GA I, 5, 204).

21. "la doctrina del derecho no es algo así como una parte de la moral, ni en absoluto una ciencia práctica, sino una ciencia puramente teórica que es para la libertad lo que la mecánica para el movimiento..., se sigue que el orden jurídico no es moral sino un mero orden natural, sobre el cual la libertad puede hacer tan poco como sobre el de la naturaleza sensible. Por eso no es sorprendente que todos los intentos de transformarlo en un orden moral se presenten como condenables por su propia absurdidad y por el despotismo en su forma más temible, su inmediata consecuencia [...]. Hay que considerar la constitución jurídica como una máquina que está previamente regulada para ciertos casos y opera por sí misma, o sea, de modo completamente ciego, tan pronto se dan estos casos; a pesar de que esta máquina está construida y regulada por manos humanas, una vez salida de la mano del artesano ha de continuar funcionando, igual que la naturaleza visible, conforme a sus propias leyes e independientemente, como si existiera por sí misma« (Schellings Werke, edición a cargo de M. Schröter, Múnich, 1927, II, p. 584; cf. pp. 582, 586).

22. "la ley debe ser una potencia; el concepto de ley [...] y el de potencia preponderante [...] deben ser reunidos sintéticamente. La ley misma debe ser el poder supremo, el poder supremo debe ser la ley, ambos deben ser una sola y misma cosa [...] Nuestro problema [...] estriba en encontrar una voluntad que sea potencia sólo cuando quiera la ley, pero que sea entonces una potencia infalible" (GA I, 3, 399-400).

23. No obstante, Fichte abona esta argumentación contractualista con alguna capa organológica. El desconcierto, no obstante, procede de la declaración de insuficiencia de lo que se presenta como autosuficiente para fundamentar el derecho político, cediendo entonces paradójicamente al organismo la optimización del mecanismo jurídico: "Este contrato de ciudadanía tiene en sí mismo el fundamento suficiente para que sea respetado, así como todo ser organizado tiene en sí mismo el fundamento completo de su ser [...]. La imagen más idónea para ilustrar este concepto es la de un producto organizado de la naturaleza. Esta imagen se ha usado con frecuencia en los últimos tiempos para describir las diversas ramas del poder público como formando una unidad, pero, que yo sepa, todavía no lo ha sido para caracterizar la relación civil entera. Al igual que en un producto de la naturaleza, cada parte puede ser lo que es sólo en esta unión y fuera de esta conexión no sería lo que es; también fuera de toda unión orgánica no sería absolutamente nada, porque sin la acción recíproca de fuerzas orgánicas que se conservan mutuamente en equilibrio, no se tendría en general ninguna forma capaz de perdurar, sino una lucha eterna entre el ser y el no-ser, que nosotros ni siquiera podemos pensar. Análogamente, sólo en la unión estatal obtiene el hombre una determinada posición en la serie de las cosas [...]. En el cuerpo orgánico, cada parte conserva continuamente al todo, y, al conservarlo, es conservada ella misma; análogamente, se relaciona el ciudadano con el Estado [...]. Cada parte -o cada ciudadano- se conserva sólo a sí mismo en la posición que le ha destinado el todo, y es justamente así como participa en la conservación del todo; y en la medida en que el todo conserva cada parte en esta su posición, el todo vuelve sobre sí mismo y propicia su autoconservación" (GA I, 4, 18-20). Aunque con un sentido diferente, en las Contribuciones recurre a la metáfora mecanicista para desacreditar al despotismo (y al llamado sistema de equilibrio que las monarquías absolutas propugnan en Europa), que reduce el hombre a una mera pieza del engranaje político. La libertad y la máquina estatal contra natura están en relación inversa (GA I, 1, 249).

24. GA I, 4, 92-93, 87 ss. Cf. Über die wissenschaftlichen Behandlungsarten, en: G.W.F. Hegel. Gesammelte Werke, edición a cargo de H. Buchner y O. Pöggeler, Hamburgo, 1968, IV, pp. 444 ss.; Differenzschrift, IV, pp. 53 ss.; System der Sittlichkeit, Hamburgo, 1967, pp. 74 ss.; System des transzendentalen Idealismus, en: Schellings Werke, II, pp. 586-587.

25. Podemos también evocar aquí las expresiones empleadas en el fragmento del systemprogramm sobre el Estado máquina: "el Estado es algo mecánico... Sólo lo que es objeto de la libertad se llama idea. ¡Por lo tanto, tenemos que ir más allá del Estado! Porque todo Estado tiene que tratar a 
hombres libres como a engranajes mecánicos [...]. Al mismo tiempo quiero sentar aquí los principios para una historia de la humanidad y desnudar hasta la piel toda la miserable obra humana: Estado, gobierno, legislación". Y más adelante emerge la intuición novedosa de "una nueva mitología", que podría engarzar con el final un tanto críptico de la NDDN: "esta mitología tiene que estar al servicio de las ideas, tiene que transformarse en una mitología de la razón". Cuando la filosofía se torne mitológica y el pueblo se vuelva racional, entonces "reinará la unidad perpetua entre nosotros. [...] Sólo entonces nos espera la formación igual de todas las fuerzas... No se reprimirá ya fuerza alguna, reinará la libertad y la igualdad universal de todos los espíritus" (G.W.F. Hegel. Escritos de juventud, F.C.E., México, 1978, pp. 219-220). Las precarias alusiones a SCHELLING DE B. STOLLBERG-RILINGER EN SU LIBRO DER STAAT ALS MASCHINE. ZUR POLITISCHEN METAPHORIK DES ABSOLUTEN FÜRSTENSTAATS (DUNCKER \& HUMBLOT, BERLÍN, 1986, PP. 188, 214, 227, 237) APENAS VAN MÁS ALLÁ DE LA SIMPLE Y RETÓRICA MENCIÓN.

26. "Puédese, inversamente, mediante una analogía con los citados fines inmediatos de la naturaleza, aclarar cierto enlace que también, empero, se encuentra más en la idea que en la realidad. Así, en una transformación total, recientemente emprendida, de un gran pueblo en un Estado, se ha utilizado muy apropiadamente la palabra organización, a menudo para designar la sustitución de magistraturas, etc., y hasta del cuerpo entero del Estado. Pues cada miembro, desde luego, debe ser, en semejante todo, no sólo medio, sino también, al mismo tiempo, fin, ya que contribuye a efectuar la posibilidad del todo, y debe, a su vez, ser determinado por la idea del todo, según su posición y su función« (Crítica del Juicio, Ak. A, V, p. 375, nota).

27. Véanse las Lecciones sobre el método del estudio académico (1802), y en particular la décima (Schellings Werke, III, pp. 328 ss.; ed. cast. Madrid, Editora Nacional, 1984): "El mundo perfecto de la Historia sería por ello mismo una naturaleza ideal, el Estado, como el organismo externo de una armonía conseguida en la libertad misma, armonía de la necesidad y la libertad. La Historia, en la medida en la que tiene la formación de esta corporación como objeto preferente, sería Historia en el estricto sentido del término" (pp. 328-329). El recurso al organismo por Schelling y Fichte NO PUEDE HACERNOS OLVIDAR QUE SUS RESPECTIVAS CONCEPCIONES DE LA NATURALEZA SON INCOMPOSIBLES -COMO ATESTIGUA EL INTERCAMBIO EPISTOLAR ENTRE AMBOS DE NOVIEMBRE DE 1800 (GA III, 4, 359 SS.)-.

\section{AUTOR}

\section{FAUSTINO ONCINA COVES}

Universitat de València 